PROCEEDINGS OF THE

AMERICAN MATHEMATICAL SOCIETY

Volume 130, Number 5, Pages 1487-1491

S 0002-9939(01)06198-6

Article electronically published on October 23, 2001

\title{
A COMBINATORIAL PROPERTY OF CARDINALS
}

\author{
PÉTER KOMJÁTH AND MIKLÓS LACZKOVICH \\ (Communicated by Carl G. Jockusch, Jr.)
}

\begin{abstract}
GCH) For every cardinal $\kappa \geq \omega_{2}$ there exists $F:[\kappa] \leq 2 \rightarrow\{0,1\}$ such that for every $f: \kappa \rightarrow[\kappa]<\omega, i<2$, there are $x, y$ such that $F(x, t)=$ $i(t \in f(y)), F(u, y)=i(u \in f(x))$.
\end{abstract}

Let $X$ be a nonempty set, and let $F$ be a coloring of $[X] \leq 2=\{H \subset X:|H| \leq 2\}$ with two colors; that is, let $F:[X]^{\leq 2} \rightarrow\{0,1\}$. If $f$ is a map from $X$ into $[X]^{<\omega}=\{Y \subseteq X: Y$ is finite $\}$, then we say that the pair $(x, y)$ is a 0-pair, if $x, y \in X, F(x, t)=0$ for every $t \in f(y)$, and $F(u, y)=0$ for every $u \in f(x)$. The definition of 1-pairs is analogous.

For an infinite cardinal $\kappa$ let $P(\kappa)$ denote the following statement. There exists a function $F:[\kappa]^{\leq 2} \rightarrow\{0,1\}$ such that for every map $f: \kappa \rightarrow[\kappa]^{<\omega}$ there exists a 0 -pair and there exists a 1 -pair.

We prove that $P(\kappa)$ fails for $\kappa \leq \omega_{1}$ (Theorem 1). We conjecture that $P(\kappa)$ is true whenever $\kappa \geq \omega_{2}$, but we can only prove this under GCH (Theorem 2). Nevertheless, our proof works in ZFC for all cardinals $\kappa$ with $\kappa^{\aleph_{2}}=\kappa$ (Theorem $3)$.

We can show that for every cardinal $\lambda$ with $\operatorname{cf}(\lambda)>\omega_{1}$ there is a cardinal preserving extension that adds a witness to $P\left(\omega_{2}\right)$ and makes $2^{\aleph_{1}}=\lambda$ (assuming GCH, Theorem 4).

Definitions and Notation. We use the standard axiomatic set theory notation and notions; see [1]. GCH stands for the Generalized Continuum Hypothesis. If $S$ is a set and $\kappa$ a cardinal, then we let

$$
[S]^{\kappa}=\{x \subseteq S:|x|=\kappa\},[S]^{<\kappa}=\{x \subseteq S:|x|<\kappa\},[S]^{\leq 2}=[S]^{1} \cup[S]^{2} .
$$

For simplicity we use the notation $F(A, B)=0$, etc. to denote that $F(x, y)=0$ holds for every $x \in A, y \in B$.

Theorem 1. $P(\kappa)$ fails for $\kappa \leq \omega_{1}$.

Proof. Assume that we are given $F:[\kappa]^{\leq 2} \rightarrow\{0,1\}$. We will find a function $f: \kappa \rightarrow[\kappa]^{<\omega}$ either with no 0 -pairs or else with no 1 -pairs.

Case 1. $\kappa=\omega$.

If there is an element $a$ such that $F(x, a)=1$ holds for every $x$, we can choose $f(x)=\{a\}$ (for $x<\omega$ ), this $f$ has no 0-pairs. We can assume, therefore, that

Received by the editors June 27, 2000 and, in revised form, November 8, 2000.

2000 Mathematics Subject Classification. Primary 03E05.

Both authors were supported by Hungarian Research Grant FKFP 2007/1997.

(C)2001 American Mathematical Society 
for every $x<\omega$ the element $g(x)$ satisfies $F(x, g(x))=0$. Now the choice $f(x)=$ $\{g(0), \ldots, g(x-1)\}$ witnesses the failure of $P(\omega)$, that is, this function has no 1-pair as for $x<y$ we have $g(x) \in f(y)$ and $F(x, g(x))=0$.

Case 2. $\kappa=\omega_{1}$.

If, for every $\alpha<\omega_{1}$, there is some $h(\alpha)<\omega_{1}$ with $F(\alpha,\{h(\alpha)\})=1$ (that is, $F(\beta, h(\alpha))=1$ holds for every $\beta<\alpha)$, then the function $f(\alpha)=\{h(\alpha)\}$ has no 0 -pair. We can assume, therefore, that there exist a countable set $X=\left\{\gamma_{0}, \gamma_{1}, \ldots\right\}$ such that for every $\alpha \in \omega_{1}-X$ there is some $\gamma_{n}$ with $F\left(\alpha, \gamma_{n}\right)=0$. Decompose $\omega_{1}-X$ as $\omega_{1}-X=X_{0} \cup X_{1} \cup \cdots$ where

$$
X_{n}=\left\{\alpha \in \omega_{1}-X: F\left(\alpha, \gamma_{n}\right)=0\right\} .
$$

Further, let $Y_{n}=X_{n}-\left(X_{0} \cup \cdots \cup X_{n-1}\right)$. Again, we can assume, by the argument at the beginning of the proof, that for every $x$ there is some $g(x)$ with $F(x, g(x))=0$. Define for $\alpha \in Y_{n} \cup\left\{\gamma_{n}\right\}$

$$
f(\alpha)=\left\{\gamma_{0}, \ldots, \gamma_{n}, g\left(\gamma_{0}\right), \ldots, g\left(\gamma_{n}\right)\right\} .
$$

This $f$ has no 1-pair.

Theorem $2(\mathrm{GCH})$. $P(\kappa)$ holds for every $\kappa \geq \omega_{2}$.

Proof. We first consider the case $\kappa=\omega_{2}$.

For $S \subseteq[\omega]^{\aleph_{0}}, j<2$ let $T_{j}(S) \subseteq \omega_{2}$ be a stationary set such that $T_{j}(S)$ and $T_{j^{\prime}}\left(S^{\prime}\right)$ are disjoint if either $S \neq S^{\prime}$ or $j \neq j^{\prime}$. (Exist as by GCH $\aleph_{2}^{\aleph_{0}}=\aleph_{2}$ and by Solovay's theorem.)

Enumerate the systems consisting of $\aleph_{1}$ disjoint finite subsets of $\omega_{2}$ as

$$
\left\{\left\{A_{i}^{\alpha}: i<\omega_{1}\right\}: \alpha<\omega_{2}\right\} .
$$

We require that $\sup \left(\bigcup\left\{A_{i}^{\alpha}: i<\omega_{1}\right\}\right)<\alpha$ by formally allowing that the systems are not defined for some values of $\alpha$.

We define $F(x, \alpha)$ by transfinite recursion on $\alpha$ (for the values $x<\alpha$ ).

Assume that $F(x, \beta)$ is defined for $x<\beta<\alpha$. If $\alpha \in T_{j}(S)$ for some $j$ and $S$, then, of course, we make $F(S,\{\alpha\})=j$. Beyond this, we make sure that the following property holds:

(*) for $\beta \leq \beta_{1}<\cdots<\beta_{n}<\alpha, j<2$, there are $\aleph_{1}$ indices $i<\omega_{1}$ that $F\left(A_{i}^{\beta},\left\{\beta_{1}, \ldots, \beta_{n}, \alpha\right\}\right)=j$.

We show that this function $F$ works.

Assume that $f(\alpha)$ is a finite subset of $\omega_{2}$. We find a 0-pair, the case of getting a 1-pair is similar.

Lemma 1. There is a countable $S$ such that for every countable $S^{\prime} \supseteq S$ there are stationary many $\alpha$ such that $F\left(S^{\prime},\{\alpha\}\right)=0$ and $f(\alpha) \cap\left(S^{\prime}-S\right)=\emptyset$.

Proof. Otherwise, for every countable $S$ there are a countable $S^{\prime} \supseteq S$ and a closed, unbounded set $C$ with the following property; if $\alpha \in C$ and $F\left(S^{\prime},\{\alpha\}\right)=0$, then $f(\alpha) \cap\left(S^{\prime}-S\right) \neq \emptyset$. We define by induction the countable sets $S_{0}, S_{1}, \ldots$ and closed, unbounded sets, $C_{0}, C_{1}, \ldots$ such that $S_{0}=\emptyset$ and for $S_{n}$ the sets $S_{n+1}, C_{n}$ are as described above. Put $S=\bigcup\left\{S_{n}: n<\omega\right\}$ and $C=\bigcap\left\{C_{n}: n<\omega\right\}$. $S$ is countable, while $C$ is closed, unbounded. Pick $\alpha \in T_{j}(S) \cap C$ (such an $\alpha$ exists as $T_{j}(S)$ is stationary). Then $f(\alpha) \cap\left(S_{n+1}-S_{n}\right) \neq \emptyset$ holds for every $n<\omega$ which is impossible, as $f(\alpha)$ is finite. 
From now on fix an $S$ as in Lemma 1.

Lemma 2. There is a countable $S^{\prime} \supseteq S$ such that if $t$ is finite, $t \cap S^{\prime}=\emptyset$, then there are stationary many $\alpha$ such that $f(\alpha) \cap \alpha \subseteq S^{\prime}$ and $F(S \cup t,\{\alpha\})=0$.

Proof. For $\alpha \in T_{0}(S), F(S,\{\alpha\})=0$. On this set, the function $f(\alpha) \cap \alpha$ is regressive, so by Fodor's lemma, there is a stationary $H \subseteq T_{0}(S)$ and a countable $S^{\prime} \supseteq S$ that $f(\alpha) \cap \alpha \subseteq S^{\prime}$ holds for $\alpha \in H$. If the statement of Lemma 2 fails, we can inductively choose the disjoint finite sets $t_{\xi}$ and closed, unbounded sets $C_{\xi}$ such that for $\alpha \in H \cap C_{\xi}$ we have $F\left(t_{\xi},\{\alpha\}\right) \neq 0$. If $C=\bigcap\left\{C_{\xi}: \xi<\omega_{1}\right\}$, then for the $\aleph_{2} \alpha \in H \cap C$ we have that $F\left(t_{\xi},\{\alpha\}\right) \neq 0$ for every $\xi<\omega_{1}$ which contradicts property $(*)$ of the construction.

Fix an $S^{\prime} \supseteq S$ as in Lemma 2.

Lemma 3. There is a finite $t$ and there are stationary many $\alpha$ such that $F\left(S^{\prime},\{\alpha\}\right)$ $=0, f(\alpha) \cap \alpha \subseteq S \cup t, f(\alpha) \cap\left(S^{\prime}-S\right)=\emptyset$.

Proof. By applying Lemma 1 to our particular pair $S, S^{\prime}$ we get stationary many $\alpha$ with the first and the last property. By Fodor's lemma, there is a finite $t$ such that for a stationary subset, the second property holds, as well.

In order to conclude the proof of $P\left(\omega_{2}\right)$ we observe that by Lemma 2 there are $\aleph_{2}$ many elements $\beta$ such that $f(\beta) \cap \beta \subseteq S^{\prime}$ and $F(S \cup t,\{\beta\})=0$. We choose a set $B$ consisting of $\omega_{1}$ of them such that the sets $\{f(\beta)-\beta: \beta \in B\}$ are disjoint. By Lemma 3 there are $\aleph_{2}$ elements $\alpha$ for which $F\left(S^{\prime},\{\alpha\}\right)=0, f(\alpha) \cap \alpha \subseteq S \cup t$, $f(\alpha) \cap\left(S^{\prime}-S\right)=\emptyset$. If $\alpha$ is one of them which is large enough, then

$$
F(f(\beta)-\beta, f(\alpha)-\alpha)=0
$$

and then $\{\alpha, \beta\}$ is a 0 -pair.

We now consider the case when $\kappa>\omega_{2}$. Let $\tau$ be an infinite, regular cardinal. Let $(H,<)$ be an ordered set of cardinality $\tau^{++}$in which $A$ is a co-initial subset of ordinal $\tau$ and $B$ is a cofinal set of ordinal $\tau^{+}$and every initial- and end-segment has cardinal $\tau^{++}$. Call a subset up-big if it has $\tau^{++}$elements in every end-segment, and down-big if it has $\tau^{++}$elements in every initial-segment. It is big if it is up-big and down-big.

Let $H^{\prime} \subseteq H$ be a big subset and $f: H^{\prime} \rightarrow[H]^{<\omega}$ a function. For $s \subseteq H, x \in H$ let $s>x$ denote that every element of $s$ is greater than $x$, and likewise for $s<x$.

Lemma 4. There is an $a \in H$ such that $\left\{x \in H^{\prime}: f(x)>a\right\}$ is up-big.

Proof. Otherwise, for every $a \in A$ there is some $b(a) \in B$ with

$$
\left|\left\{x \in H^{\prime}: x>b(a), f(x)>a\right\}\right| \leq \tau^{+} .
$$

There is a $b \in B$ with $b>b(a)$ for $a \in A$, and then $H^{\prime}$ can have only at most $\tau^{+}$ elements above $b$, a contradiction.

Lemma 5. There is a $b \in H$ such that $\left\{x \in H^{\prime}: f(x)<b\right\}$ is down-big.

Proof. Otherwise, for every $b \in B$ there is some $a(b) \in A$ with

$$
\left|\left\{x \in H^{\prime}: x<a(b), f(x)<b\right\}\right| \leq \tau^{+} .
$$

There is an $a \in A$ which assumes the value of $a(b)$ for $\tau^{+}$many $b \in B$ and we get that $H^{\prime}$ has only at most $\tau^{+}$elements below $a$, a contradiction. 
Let $X \supseteq H$ be some set of cardinal $\kappa$ and assume that either $\operatorname{cf}(\kappa)>\tau^{++}$or else $\kappa$ is singular and $\operatorname{cf}(\kappa)<\tau$. Given $\kappa$ this can be arranged by choosing either $\tau=\omega$ or $\tau=\omega_{3}$. We are going to construct a function $F:[X]^{2} \rightarrow[X]^{<\omega}$ witnessing $P(\kappa)$.

Lemma 6. There is a family of functions $f_{\alpha}: H_{\alpha} \rightarrow[X]^{<\omega}$ for $\alpha<\kappa$ such that $H_{\alpha}$ is always big and every $f: H \rightarrow[X]^{<\omega}$ restricts to some $f_{\alpha}$.

Proof. In the first case this is obvious as by the GCH and $\operatorname{cf}(\kappa)>\tau^{++}$the number of all $f: H \rightarrow[X]^{<\omega}$ functions is $\kappa$, so we can take $H_{\alpha}=H$ and let $\left\{f_{\alpha}: \alpha<\kappa\right\}$ enumerate the $H \rightarrow[X]^{<\omega}$ functions.

In the second case decompose $X$ as an increasing union $X=\bigcup\left\{X_{\xi}: \xi<\operatorname{cf}(\kappa)\right\}$ with $\left|X_{\xi}\right|<\kappa$. Assume that $f: H \rightarrow[X]^{<\omega}$. For every $a \in A$ there is some $\xi<\operatorname{cf}(\kappa)$ such that

$$
\left|\left\{x<a: f(x) \subseteq X_{\xi}\right\}\right|=\tau^{++} .
$$

For every $b \in B$ there is some $\xi<\operatorname{cf}(\kappa)$ such that

$$
\left|\left\{x>b: f(x) \subseteq X_{\xi}\right\}\right|=\tau^{++} .
$$

By cardinality considerations, there is a $\xi$ that is good for $\tau$ many $a \in A$ and $\tau^{+}$ many $b \in B$ and so $H^{\prime}=\left\{x \in H: f(x) \subseteq X_{\xi}\right\}$ is big. To finish the proof we only have to remark that given $H^{\prime}, \xi<\operatorname{cf}(\kappa)$ the number of these functions is less than $\kappa$, so we have altogether $\kappa$ many such functions.

We now describe the definition of $F$. Let $<_{w}$ be a well ordering of $H$ into order type $\tau^{++}$.

For $x, y \in H$ we set

$$
F(x, y)= \begin{cases}0, & x<y, x<_{w} y \\ 1, & x<y, y<_{w} x\end{cases}
$$

For $\alpha<\kappa$ choose the elements $y_{\alpha}, z_{\alpha} \in X-\bigcup\left\{\operatorname{Ran}\left(f_{\beta}\right): \beta \leq \alpha\right\}$ different from each other. Choose also $a_{\alpha}, b_{\alpha} \in H$ in such a way that $\left\{x \in H_{\alpha}: f_{\alpha}(x) \cap H>a_{\alpha}\right\}$ is up-big and $\left\{x \in H_{\alpha}: f_{\alpha}(x) \cap H<b_{\alpha}\right\}$ is down-big.

We now define $F$ for some further pairs:

$$
\begin{aligned}
& F\left(x, y_{\alpha}\right)= \begin{cases}0, & x \in f_{\alpha}(z), z \in H_{\alpha}, f_{\alpha}(z) \cap H>a_{\alpha}, \\
0, & x>a_{\alpha}, x \in H, \\
1, & x<a_{\alpha}, x \in H ;\end{cases} \\
& F\left(x, z_{\alpha}\right)= \begin{cases}0, & x>b_{\alpha}, x \in H, \\
1, & x \in f_{\alpha}(z), z \in H_{\alpha}, f_{\alpha}(z) \cap H<b_{\alpha}, \\
1, & x<b_{\alpha}, x \in H .\end{cases}
\end{aligned}
$$

If $x$ is an element of $X-H$ different from all the points $y_{\alpha}, z_{\alpha}$, we choose arbitrarily a $u \in H$ and set $F(y, x)=0$ for $y \in H, y>u$, and $F(y, x)=1$ for $y \in H, y \leq u$. So far, we have defined $F(x, y)$ if $x \in H$ and $y \in X$, and for some other pairs, as well. For the remaining pairs we can extend $F$ arbitrarily. We notice that for every $x \in X-H, F(x, y)=0$ if $y \in H$ is large enough and $F(x, y)=1$ if $y \in H$ is small enough. Moreover, for every $x \in X$ for all but $\tau^{+}$elements $y \in H$ it is true that if $y$ is large enough, then $F(x, y)=0$, and if it is small enough, then $F(x, y)=1$.

Assume now that $f: X \rightarrow[X]^{<\omega}$. There is some $\alpha<\kappa$ that $f \mid H_{\alpha}=f_{\alpha}$. All but $\tau^{+}$many large enough $x \in H_{\alpha}$ have $F\left(x, f\left(y_{\alpha}\right)\right)=0$. If such an $x$ has even $f_{\alpha}(x) \cap H>a_{\alpha}$, then we also have that $F\left(f(x), y_{\alpha}\right)=0$ and we are done. 
Theorem 3 (ZFC). $P(\kappa)$ holds if $\kappa^{\aleph_{2}}=\kappa$.

Proof. In the above proof (for $\kappa>\aleph_{2}$ ) we needed an instance of GCH in Lemma 6 to show that for $|H|=\aleph_{2},|X|=\kappa$ we have no more than $\kappa$ functions from $H$ to $[X]^{<\omega}$. But the number of these functions is $\kappa^{\aleph_{2}}$.

As mentioned in the introduction, we could not establish $P\left(\omega_{2}\right)$ in ZFC alone. We can, nevertheless, show that $P\left(\omega_{2}\right)$ can consistently hold with any reasonable value of $2^{\aleph_{1}}$.

Theorem $4(\mathrm{GCH})$. Assume that $\operatorname{cf}(\lambda)>\omega_{1}$. Then it is consistent that $2^{\aleph_{1}}=\lambda$ and $P\left(\omega_{2}\right)$ holds.

Proof. With a preliminary forcing we can assume that $C H$ and $2^{\aleph_{1}}=\lambda$ already hold in the ground model. We add a "generic" coloring $F:\left[\omega_{2}\right] \leq 2 \rightarrow\{0,1\}$ and show that it works.

Let $p \in P$ if it is of the form $p=(s, h)$ where $s \in\left[\omega_{2}\right] \leq 2, h:[s]^{2} \rightarrow\{0,1\}$. Extension is defined as $p^{\prime}=\left(s^{\prime}, h^{\prime}\right) \leq p=(s, h)$ if $s^{\prime} \supseteq s, h=h^{\prime} \mid[s] \leq 2$. If $G$ is a generic filter, then we let $F=\bigcup\{h:(s, h) \in G\}$. We claim that $F$ witnesses $P\left(\omega_{2}\right)$.

Assume that $1 \Vdash f: \omega_{2} \rightarrow\left[\omega_{2}\right]^{<\omega}$. We show that there is a 0 -pair (the other case is similar). The following argument is in $V[G]$.

Claim. There exists $T \in\left[\omega_{2}\right]^{\aleph_{0}}$ such that for every $T^{\prime} \in\left[\omega_{2}\right]^{\aleph_{0}}$ with $T^{\prime} \supseteq T$ there is some $\alpha<\omega_{2}$ such that $F\left(T^{\prime}, \alpha\right)=0$ and $f(\alpha) \cap\left(T^{\prime}-T\right)=\emptyset$.

Proof of Claim. Assume otherwise. Then, for every countable $T$ there is some countable $T^{\prime} \supseteq T$ such that whenever $F\left(T^{\prime}, \alpha\right)=0$ then necessarily $f(\alpha) \cap\left(T^{\prime}-T\right) \neq$ $\emptyset$. Define inductively $T_{0} \subseteq T_{1} \subseteq \cdots$ by $T_{0}=\emptyset, T_{n+1}=T_{n}^{\prime}$. Set $T_{\omega}=\bigcup\left\{T_{n}: n<\right.$ $\omega$. We eventually get that if $F\left(T_{\omega}, \alpha\right)=0$ then $f(\alpha) \cap\left(T_{n+1}-T_{n}\right) \neq \emptyset$ holds for $n=0,1, \ldots$ which contradicts the finiteness of $f(\alpha)$. And indeed such an $\alpha$ exists, as we can easily force it.

Assume therefore that $p \Vdash T$ is as in the statement of the Claim. For every $\alpha<\omega_{2}$ let $p_{\alpha} \leq p$ be a condition forcing that $F(T, \alpha)=0$. Applying the $\Delta$-system lemma we find $\aleph_{2}$ conditions $\left\{p_{\alpha}: \alpha \in Z\right\}$ such that $p_{\alpha}=\left(T^{\prime} \cup T_{\alpha}, h_{\alpha}\right)$ where $T^{\prime} \supseteq T$, and $h_{\alpha} \mid\left[T^{\prime}\right] \leq 2=h$. By the Claim there is some $\beta$ such that $F\left(T^{\prime}, \beta\right)=0$ and $f(\beta) \cap\left(T^{\prime}-T\right)=\emptyset$, moreover, this is forced by some $\bar{p}=(\bar{s}, \bar{h}) \leq\left(T^{\prime}, h\right)$. For some $\alpha \in Z$ we have $T_{\alpha} \cap \bar{T}=\emptyset$ and we can consider $q=\left(\bar{T} \cup T_{\alpha}, h^{\prime}\right)$ where $h^{\prime}$ is 0 on $T_{\alpha} \times\left(\bar{T}-T^{\prime}\right)$. Then $q \leq \bar{p}, p_{\alpha}$ and $(\alpha, \beta)$ will be a 0 -pair as $f(\alpha) \subseteq T \cup T_{\alpha}$ and $f(\beta) \subseteq\left(\left(T^{\prime}-T\right) \cup\left(\bar{T}-T^{\prime}\right)\right)$.

We notice that even this can slightly be extended to show that if $\kappa \leq \lambda, \operatorname{cf}(\kappa)>$ $\omega, \operatorname{cf}(\lambda)>\omega_{1}$, then it is consistent that $2^{\aleph_{0}}=\kappa, 2^{\aleph_{1}}=\lambda$, and $P\left(\omega_{2}\right)$ holds. One only has to add $\kappa$ many Cohen reals simultaneously.

\section{REFERENCES}

[1] T. Jech, Set Theory, Academic Press, 1978. MR 80a:03062

Department of Computer Science, Eötvös University, P.O. Box 120, 1518, Budapest, HUNGARY

E-mail address: kope@cs.elte.hu

Department of Analysis, Eötvös University, P.O. Box 120, 1518, Budapest, Hungary

E-mail address: laczk@cs.elte.hu 